\title{
Selected Major Issues in Vaccine Safety
}

\author{
John K. Iskander ${ }^{a}$ Jane Gidudu ${ }^{a}$ Nelson Arboleda ${ }^{a}$ Wan-Ting Huang ${ }^{b}$ \\ ${ }^{a}$ Immunization Safety Office, Office of the Chief Science Officer, and ${ }^{b}$ Epidemic Intelligence Service, Office of \\ Workforce and Career Development, US Centers for Disease Control and Prevention, Atlanta, Ga., USA
}

\section{Key Words}

Immunization • Vaccine safety • Post-licensure surveillance

\begin{abstract}
Recent examples of the major public health benefits of vaccination include global reductions in measles mortality and record low levels of vaccine-preventable diseases in the United States. Nevertheless, real or perceived vaccine safety issues may adversely impact vaccine programs. Robust postlicensure safety monitoring which combines active and passive surveillance with use of standardized case definitions for adverse events is the scientific basis for assessing safety concerns. Emerging aspects of vaccine safety science include clinical research networks and vaccine risk communication research. Current high-profile safety issues include the introduction of 2 second-generation rotavirus vaccines, for which close monitoring of intussusception is necessary. To date, data from the US do not indicate an elevated risk associated with the licensed Merck vaccine (Rotateq ${ }^{\circledR}$ ). An issue of global interest is the use of thimerosal as a preservative in multi-dose vaccine vials. Comprehensive independent reviews as well as recently published research have reaffirmed the lack of association between thimerosal and neurodevelopmental disorders, including autism. Expanded use of annual influenza vaccine and pandemic planning in the developed or developing world should include plans for safety monitoring. As the number of newly licensed vaccines increases, potentially preventable vaccine administration er-
\end{abstract}

rors and post-vaccination events such as syncope are being increasingly recognized. Primary care clinicians and others involved in giving vaccines should follow proper vaccine storage, handling and administration procedures and should participate in adverse event following immunization (AEFI) reporting systems. The Brighton Collaboration provides another outlet, which interested clinicians and researchers can participate in, increasing the global vaccine safety knowledge base. Increased knowledge of and participation in vaccine safety systems at all levels of health care systems in both developed and developing country settings will allow vaccines to maintain their excellent safety track record, as safety data is used to improve immunization practice.

Copyright $\odot 2008$ Nestec Ltd., Vevey/S. Karger AG, Basel

\section{Background}

\section{Global Benefits of Vaccines}

Vaccination programs are considered to be among the most beneficial and cost-effective public health interventions [1-7]. Many established vaccine programs are actually cost saving when viewed from a societal perspective $[8,9]$. Recently published data have provided additional examples of how vaccines can reduce morbidity and mortality in both developed and developing countries. Roush and colleagues [10] observed record or near-record lows in vaccine-preventable diseases reported in the United States (US) in 2006, and the global measles mortality re-

\section{KARGER \\ Fax +4161306 1234 \\ E-Mail karger@karger.ch}

www.karger.com (c) 2008 Nestec Ltd., Vevey/S. Karger AG, Basel

0517-8606/08/0662-0093\$24.50/0

Accessible online at:

www.karger.com/ane
John Iskander, MD, MPH

Acting Director, Immunization Safety Office, Office of the Chief Science Officer Centers for Disease Control and Prevention

1600 Clifton Road MS D-26, Atlanta, GA 30333 (USA)

Tel. +1 404639 8889, Fax +1 404639 8834, E-Mail jxi0@cdc.gov 
duction initiative using measles-containing vaccine as a central tool has exceeded its target goals [11]. Vaccination programs may have additional benefits to those not targeted for vaccination as demonstrated by decreased carriage of pneumococcus and subsequent decreased disease levels in age groups not targeted for vaccination [12].

\section{'Lifecycle' of Vaccine Safety Concerns}

Maximizing the benefits of vaccines requires relatively high and sustained coverage (uptake) levels, so that herd immunity thresholds are achieved and susceptible individuals do not accumulate $[13,14]$. Paradoxically, it is just when vaccine benefits are most apparent and vaccine coverage is highest that vaccine safety concerns are most likely to arise in the consciousness of the general public and the media [15]. These concerns may arise partly because patients, parents and health care providers no longer have any first-hand experience with vaccine-preventable diseases. Thus, it is often said that vaccines are the victims of their own success. A pattern that has been observed in several countries, including Japan, the United Kingdom (UK) and Nigeria, where loss of public confidence in vaccines was followed by decreased coverage leading to outbreaks of vaccine-preventable diseases, often with considerable morbidity and mortality [16]. It is only after such first-hand experiences that confidence in vaccination resumes and coverage rebounds. This article describes a proactive strategy to monitor vaccine safety on an ongoing basis in order to maintain both the public and health care provider confidence in the safety of vaccines.

Because of the global scope of vaccines and vaccination programs, this review will discuss overall considerations for monitoring vaccine safety and will highlight several key issues as case examples. A comprehensive review of all issues related to vaccine safety methodologies and specific issues under study is beyond the scope of this paper. Similarly, although monitoring of safety within vaccine clinical trials is crucial, this review will not address pre-licensure study of safety in detail. Information resources, including websites, which provide updated information on newly emerging issues in vaccine safety will be highlighted.

\section{Post-Licensure Safety Surveillance}

The primary rationale for closely monitoring the safety of newly licensed vaccines and newly introduced vaccine programs is that clinical trials do not have the power to detect rare vaccine adverse events. Even the very largest trials [17-19] which have enrolled up to 70,000 participants cannot detect vaccine adverse events rarer than 1 in 10,000 vaccinees. Although rare side effects may halt vaccination programs on occasion $[20,21]$, providers should understand that some commonly used vaccines have demonstrated rare and potentially serious side effects; in these instances, policymaking bodies have judged that the individual and community benefits of vaccination outweigh the risks. For instance, measles-containing vaccines which have played a key role in measles mortality reduction are associated with thrombocytopenia (which is self-limited and uncomplicated) in approximately 1 in 30,000 vaccinees [22]. As with any medical product, safety considerations for vaccines should be rigorously scientifically assessed; however, policy decisions must take into account both documented benefits and risks.

In some instances, adverse reactions to vaccines may be so rare that they only emerge after extensive post-licensure use. The association of myopericarditis with the NYC BOH strain of smallpox vaccine in approximately 1 per 10,000 vaccinees was discovered long after global smallpox eradication was achieved, when the US undertook military and civilian smallpox vaccination programs in 2003 as part of bioterrorism preparedness activities [23]. This was due partly to the rareness of the finding but also because of the much more active safety monitoring measures undertaken in the absence of wild disease [21].

Another key reason for post-licensure monitoring is that trials, regardless of their size, may exclude certain populations [24] such as persons with underlying medical conditions or premature infants. Because vaccination recommendations, particularly for children, are often universal, the public health community has a duty to ensure the safety of new vaccines under actual conditions of everyday use and practice among diverse patient populations. Like other licensed medical products, vaccines may be subject to use outside of FDA-recommended age or risk group indications. This is referred to as 'off-label' use; monitoring off-label vaccine usage can be an important aspect of safety monitoring [25].

\section{Methods}

\section{Vaccine Safety Monitoring Systems}

Adverse event reporting systems for vaccines, often referred to as spontaneous reporting systems (SRS), are one of the primary ways by which vaccine safety is monitored. This paper will discuss the objectives of such systems as well as their strengths and weaknesses. Reporting sus- 
pected adverse reactions to vaccines to SRS is one of the key ways in which frontline healthcare providers can participate in improving the safety of vaccines. All suspected reactions are referred to as adverse events following immunization (AEFI), which does not imply a causal relationship to vaccination is known or suspected. Often such passive surveillance systems are complemented by active or enhanced surveillance, which does not depend on reporting from individual practitioners and which provides information on both vaccinated and unvaccinated persons and populations.

With rare exceptions, most individual case reports of vaccine adverse events cannot be definitely ascribed to vaccines, nor can a vaccine be definitively ruled out as the cause. The simple finding that an event occurred after vaccination is not sufficient to indicate a 'cause and effect' relationship. Parents and patients will not always agree with this reasoning, underscoring the importance of frontline vaccine providers both knowing the basic aspects of vaccine safety science and having strong communication and empathy skills. Epidemiological studies that show elevated risks of events in vaccinated compared with unvaccinated individuals, especially if the data indicate a specific period of risk following vaccination or a biologically plausible association, provide relatively strong evidence of causality [26]. These studies are often conducted using 'large linked databases', an active surveillance model which combines information on patient vaccination status, demographics and medical outcomes; the US Vaccine Safety Datalink [27] is one prominent example.

\section{Role of Advisory Bodies and Expert Panels}

One of the most important reasons for comprehensive vaccine safety monitoring is to ensure that immunization policies and recommendations use the best data available to promote use of vaccines in a safe and effective manner. Providers should be aware of and follow recommendations for use of vaccines set forth by the advisory groups within their country of practice. The US Advisory Committee on Immunization Practices recently revised the general immunization recommendations [28] and also publishes and posts online guidelines for use of vaccines in the U.S. [29]. Globally, the Global Advisory Committee on Vaccine Safety (GACVS) of the World Health Organization (WHO) regularly reviews, summarizes and publishes information regarding the safety of both new and established vaccines $[6,30]$.

For particularly difficult or controversial issues involving vaccine safety, independent panels may be con- vened to comprehensively review evidence and systematically provide guidance to policy-making bodies and the public health and medical community, as well as recommendations for further research. The US Institute of Medicine (IOM) [31] has issued numerous reports concerning vaccine safety over the past 2 decades [32], but other systematic evidence reviews such as those of the Cochrane Collaboration [33] fall into this category as well. Expert meetings may also be convened, such as that held by the US National Vaccine Program Office at which evidence for a causal relationship between the first rotavirus vaccine (Rotashield ${ }^{\circledR}$ ) and intussusception was reviewed [34].

\section{Standardized Case Definitions and the Brighton Collaboration}

Vaccines are used worldwide, and a shared terminology in the field of vaccine safety is essential. Standardization of AEFI reporting facilitates comparability and communication of vaccine safety data, which can play a key role in maintaining trust in current immunization programs.

Unlike vaccine safety, safety cannot be measured directly. Safety can only be inferred from the relative absence of vaccine adverse events. The lack of standard case definitions and guidelines for vaccine adverse events has hindered our ability to compare vaccine safety data. Comparability is important for an evidence-based understanding of the safety of different vaccines and vaccines used in different populations. However, relatively little work to develop case definitions for use in immunization safety has occurred $[35,36]$ before the establishment of the Brighton Collaboration.

\section{The Brighton Collaboration}

Work began with the formation of a steering committee and creation of working groups, composed of international volunteers with expertise in vaccine safety, patient care, pharmaceuticals, regulatory affairs, public health and vaccine delivery. The guidelines for collecting, analyzing and presenting safety data facilitate sharing and comparison of vaccine data from different geographic locations among vaccine safety professionals worldwide.

The Brighton Collaboration, in concert with the WHO and the US and European Centers for Disease Control and Prevention (CDC and ECDC), has been working to develop and disseminate standardized case definitions for AEFI. The case definitions are categorized by the levels of evidence available, which will differ according to whether it is gathered in prospective 
clinical trials or passive post-marketing surveillance, or whether it occurs in a developed or developing country [37-42].

The Brighton Collaboration has enlisted volunteer experts from around the world to define common signs and symptoms such as fever [43] and rash [44], as well as less common clinical entities such as aseptic meningitis [45] and intussusception [46]. A complete list is available and can be downloaded via a quick registration process at http://www.brightoncollaboration.org/internet/en/in$\mathrm{dex} / \mathrm{html}$. Finalized definitions are published in the journal Vaccine and, as of November 2007, a total of 22 case definitions have been completed and published.

This work constituted the first structured effort to build international consensus on a 'common language' needed to further scientific understanding of vaccine safety. Today, the use of the Brighton Collaboration case definitions is recommended by key organizations in vaccine safety including WHO, the IOM, the Food and Drug Administration (FDA) and the European Medicines Agency (EMEA) [32, 47-49]. In order to enhance the quality of submitted reports, CAEFISS (the Canadian Adverse Event following Immunization Surveillance System) has incorporated specific Brighton definitions into its reporting structure (http://www.phac-aspc.gc.ca/im/ pdf/hc4229e.pdf). The Collaboration with its more than 1,500 voluntary professional participants originating from over 90 countries is unique in its capacity.

Developing globally accepted case definitions is one aspect of vaccine safety standardization. New efforts to explore standardization beyond case definitions would need to look at the various aspects of data such as coding, collection, analysis, as well as vaccine safety study designs. Although discussion of these initiatives is beyond the scope of this paper, interested readers are referred to the following references [50-52].

\section{The Proposed Global Vaccine Safety DataNet}

Historically, most vaccines have been developed, produced and first introduced in countries (primarily in North America and Europe) with considerable resources for evaluating safety in both clinical trials and post-marketing surveillance. However, vaccine manufacturing is globalizing, with production in Brazil, China, India and other countries. Some new vaccines, wherever they are manufactured, are increasingly being introduced first in developing countries that lack extensive infrastructure for monitoring safety. The most recent example of this is the rotavirus vaccine; one version (Rotarix ${ }^{\circledR}$, GlaxoSmithKline) has been introduced in- to the developing world before introduction in the US or Europe.

Besides the Brighton Collaboration, there is another proposed global approach to evaluating vaccine safety. A Global Vaccine Safety DataNet meeting was held in Annecy, France, in September 2007. Several developed countries, such as the US, UK and Denmark [53], already have large linked databases (LLDB) to track vaccinations and clinical outcomes within their countries. Computer databases and technology exist in other countries which would allow the development of a Global Vaccine Safety DataNet. At this meeting, emphasis was placed on the concept of using existing LLDB to promote rapid identification of possible causal relationships between adverse events and associated vaccine(s). The conference had 3 main objectives [Steve Black, pers. commun.]: (1) to allow evaluations of safety concerns across a large combined population in the US and the European Union that would provide enhanced statistical power compared with that available in any one country alone, (2) to allow comparison of results obtained from a study in one country with a readily available alternative population, and (3) to provide the opportunity for sites in the developed world to mentor and facilitate development of expertise in the developing world. The eventual goal would be to have a global network with sufficient representation, experience and size to allow vaccine safety concerns to be addressed in a comprehensive, rapid and locally credible manner.

This network would foster standardized implementation of Brighton-developed case definitions using existing computerized data within countries and could form the basis for collaborative studies across several countries. Establishment of a Global Vaccine Safety DataNet would greatly expand the geographic scope of current vaccine safety capacity. It would also allow results obtained in one country or region to be tested in additional populations, as well as performance of studies in larger populations to provide additional statistical power for identifying rare adverse events. Furthermore, the development of data networks in locations that currently lack them will facilitate the local evaluation of safety issues or hypotheses in populations around the world. This will be critical for vaccines in development (such as malaria and tuberculosis) that may be introduced in the developing world but will not be used in the developed world, and may also provide valuable information on the potential risk of adverse events whose occurrence rates differ by location, or where the endemic rates of the disease may affect the results of risk-benefit analyses. 


\section{Clinical Vaccine Safety Research}

Although serious adverse events following vaccines are rare, they are concerning to patients or their parents as well as vaccine providers, who may face complex medical decision making regarding future vaccinations after an AEFI. Several countries, including Australia and Italy $[54,55]$, have undertaken clinical immunization consultation services in order to provide expert medical evaluation and guidance for patients who have experienced a vaccine adverse event. Often reimmunization can be safely completed with close medical oversight and monitoring [56]. In the US, the Clinical Immunization Safety Assessment Network is a network of 6 medical research centers with expertise in immunization safety conducting clinical research on immunization-associated health risks. The Clinical Immunization Safety Assessment Network is involved in the design and conduct of research on adverse events and individual variation and seeks to supply providers and the public with evidence-based guidelines when evaluating adverse events or considering vaccination of those at risk of adverse events. The Network seeks to study the pathophysiologic basis of AEFI, as well as risk factors (including genetic host factors) associated with developing AEFI [57]. Current research protocols include evaluation of Guillain-BarréSyndrome, temporally associated with vaccination, development of algorithms and guidelines for hypersensitivity reactions to vaccines, and observation of children with certain immune deficiencies who receive live virus vaccines [58].

\section{Specific Vaccine Safety Issues of Interest}

\section{Introduction of New Vaccines with Potential Safety}

\section{Concerns: The Rotavirus Example}

In 1999, the first rotavirus vaccine licensed in the US (Rotashield) was hailed as a public health breakthrough, with the potential to decrease the substantial morbidity and mortality caused by rotavirus infections in the developing world. However, within 4 months of publication of the official recommendation of the vaccine [59], intussusception [46], a potentially serious form of intestinal obstruction, was identified as a safety concern emerging from the Vaccine Adverse Event Reporting System (VAERS), the US SRS. A subsequent controlled study within the Vaccine Safety Datalink confirmed a statistically significant elevated risk of intussusception within 3-7 days after the first dose; in October 1999, the recommendations of the Advisory Committee on Immunization Practices for the product were withdrawn [60] and the manufacturer voluntarily withdrew the vaccine. Despite lingering controversy, the final scientific judgment of the public health community on Rotashield was that it was associated with 1 additional case of intussusception per 10,000 vaccine recipients [34].

However, the public health need for safe and effective rotavirus vaccines that can be used globally remained. The year 2006 saw the publication of large trials involving newer-generation rotavirus vaccines $[18,19]$ and the licensure of Merck's Rotateq ${ }^{\circledR}$ vaccine in the US. Neither pre- nor post-licensure monitoring to date [61] have indicated a causal association between Merck's product and intussusception. GSK's product Rotarix has been licensed in Mexico and elsewhere, and to date, has been shown to have a similarly acceptable safety profile as the Merck vaccine [19]. In mid 2007, concerns arose regarding prelicensure reports of Kawasaki disease following Rotateq. Biological evidence for such an association is very limited and involves natural rotavirus infection rather than vaccine strains [62]. The Global Advisory Committee on Vaccine Safety recently reviewed available data and found no convincing evidence for a causal association between licensed rotavirus vaccines and Kawasaki disease [30], although active and passive post-licensure study is ongoing.

\section{Preservative-Containing Vaccines and Thimerosal}

In 1999, a group of US federal agencies recommended that vaccine manufacturers take steps to decrease the amount of thimerosal (also referred to as thiomersal or merthiolate), a mercury-containing preservative, contained in routine childhood vaccines [63]. With the exception of some formulations of influenza vaccine, childhood vaccines in use in the US were essentially thimerosal free as of late 2001 or early 2002 [64]. An initial IOM report hypothesized that thimerosal could possibly be linked to neurotoxic effects. However, in 2004, the IOM conducted a comprehensive review of the issue of vaccines and autism and concluded that well-conducted studies supported the lack of any such association and that research in autism should move in other directions [65]. This view has been supported by recently published reviews [66]. Newly published studies [67] using standardized developmental testing and careful controlling of possible confounders continue to support thimerosal's lack of neurotoxicity. The issue of thimerosal continues to be monitored by GACVS because preservative-containing multi-dose vials of vaccine are still widely used around the world, especially in settings where inadvertent contamination of vaccines remains a major concern. 
Where both preservative-free and preservative-containing vaccines are available, official recommendations, such as those of the U.S. ACIP for influenza vaccines [68], do not express a preference for use of one type of vaccine over another because of a lack of scientific evidence of harm caused by low dose of thimerosal.

Providers should also be aware of earlier work conducted by Andrew Wakefield in the UK suggesting a link between the measles-mumps-rubella vaccine and autism [69]. These findings have not been confirmed by multiple controlled studies and expert reviews which followed [70, 71]. In light of both scientific and ethical concerns regarding Wakefield's original work, most of the studies' original authors have withdrawn their interpretation of the original findings [72].

\section{Safety of Seasonal (Annual) and Pandemic Influenza} Vaccines

Seasonal (annual) influenza is commonly viewed by health professionals and the general public as a predictable and controllable illness. Because of its routine/seasonal occurrence, its significant morbidity and mortality are often underappreciated. Individuals such as the elderly and younger children, and people with certain health conditions (such as asthma, diabetes or heart disease), are at high risk of serious complications of influenza. Every year in the US, $5-20 \%$ of the population acquire influenza; more than 200,000 people are hospitalized from its complications and 30,000-50,000 people die. In addition, one study estimated that 250,000500,000 people worldwide die from complications of influenza [73].

It is fundamental to view influenza as a global disease in regard to both seasonal outbreaks and the looming threat of pandemic influenza. The pathogen that will produce the next influenza pandemic is unpredictable, but the H5N1 virus is a candidate. Since 1997, when the first human case of $\mathrm{H} 5 \mathrm{~N} 1$ infection was reported, outbreaks have remained sporadic, with person-to-person transmission being uncommon. However, its mortality rate has been significant at $60 \%$ of human infections. Preparations for a possible pandemic due to an $\mathrm{H} 5 \mathrm{~N} 1$ virus have included development of stockpiled influenza vaccines against $\mathrm{H} 5 \mathrm{~N} 1$; in April 2007, the FDA approved the first vaccine against $\mathrm{H} 5 \mathrm{~N} 1$ avian influenza for use in humans [74]. Although H5N1 represents the greatest current pandemic threat, vigilance for the emergence of other viruses with pandemic potential is critical. The basic 3 -fold strategy for an influenza pandemic expands upon the seasonal influenza response: delaying the onset of the epidemic, slowing the transmission of the virus, and lastly, reducing transmission rates to decrease the overall number of infections and resulting deaths.

Vaccination in the context of an influenza pandemic will likely take place over many months and involve a large number of individuals. A pandemic influenza vaccination campaign must contain a vaccine safety monitoring plan which will be carried out throughout all phases of the pandemic response [75]. There are several factors related to a pandemic influenza vaccine response that make vaccine safety monitoring particularly important. The scale of the response will require the ability to monitor the substantial surge of reporting of adverse events; the diversity of populations to be vaccinated (e.g., age, gender, pre-existing health conditions, pregnancy) may result in the occurrence of unexpected rare and serious vaccine-associated adverse events, and the timeliness of the response may require expedited pre-licensure safety testing of the vaccine that will make post-licensure monitoring even more critical. Continuous monitoring of prepandemic and pandemic vaccines to assess immunization benefits and risks will be essential in ensuring vaccine safety during a pandemic. While serious vaccine side effects are rare, it is difficult to detect them in clinical trials that are conducted on vaccines before they are licensed. The CDC, along with its public health partners and the US FDA, will play a vital role in assuring vaccine safety.

Global collaboration to monitor influenza has significantly improved and has accelerated substantially in response to the outbreaks of the H5N1 avian influenza virus and the epidemic of the coronavirus responsible for the severe acute respiratory syndrome that occurred in 2003 [73]. Global public health initiatives to rapidly identify outbreaks of infectious diseases are expected to slow or modify, but not prevent the next influenza pandemic. The challenge in a pandemic preparation is to sustain public interest and the interest of the private and public organizations critical to initiatives that must be prepared now to plan for a pandemic that may not occur for many years. Within vaccine safety, preparedness activities need to focus on establishing systems that will have the substantial surge capacity to monitor vaccine adverse events.

It is estimated that in order to develop, test and manufacture 300 million doses of vaccine for the US, it would take 9-12 months, although an important goal is for the nation to be able to produce 300 million courses within 6 months by the year 2011 by using cell-based technology (which is not yet licensed). During both the pre-pandemic and early pandemic vaccination periods, vaccine 
is anticipated to be scarce and demand will greatly exceed supply. Vaccination efforts will be targeted to priority groups, and monitoring of adverse events will be accomplished utilizing a multifaceted approach to evaluate serious adverse events as well as specific studies (subpopulations and vulnerable populations) to determine whether a particular adverse event is caused by a specific vaccine. The most critical component of a vaccine safety monitoring plan is to ensure the appropriate capacity to monitor vaccine safety in a pandemic influenza response. This includes assessment and enhancement of the current vaccine safety infrastructure to provide the capacity for rapid and ongoing analyses within the parameters of individual privacy and confidentiality rights [76].

To mitigate the substantial morbidity and mortality associated with seasonal influenza, comprehensive approaches to seasonal immunization have been implemented along with surveillance and treatment strategies. During a severe influenza pandemic without effective control measures, it is estimated that up to 9 million hospitalizations and nearly 2 million deaths may result. Facilitating the manufacture, distribution, and administration and monitoring of safe and effective pandemic influenza vaccines are key components of pandemic preparedness activities.

\section{Potentially Preventable Adverse Events}

Vasovagal syncope, a transient loss of postural tone and consciousness because of abnormal sympathetic reflex with spontaneous recovery, has been observed after medical procedures [77] including vaccination. In the US, post-vaccination syncope reports have been received and analyzed by VAERS [78], a passive post-licensure surveillance system jointly operated by the CDC and the FDA. A summary of VAERS data during 1990-1995 involving 697 cases of syncope occurring within $12 \mathrm{~h}$ after vaccination has revealed that $45.4 \%$ were aged $10-19$ years and $57.5 \%$ were females. For those with syncope onset within $1 \mathrm{~h}$ after vaccination, $63.2 \%$ were within 5 min and $88.8 \%$ were within 15 min after vaccination [79].

Injury related to post-vaccination syncope rarely occurs but can be life threatening. A review describing 107 reports of post-vaccination syncope-induced falls indicated that 68 (63.6\%) events have resulted in secondary injuries, including 1 fatality from intracranial hemorrhage in an adolescent boy aged 15 years [80]. To prevent injury from post-vaccination syncope, the Advisory Committee on Immunization Practices of the CDC currently recommends that 'vaccine providers should strongly consider observing patients for $15 \mathrm{~min}$ after they are vaccinated. If syncope occurs, patients should be observed until the symptoms resolve' [28]. This recommendation applies to all ages and all vaccines. Additional research is needed to systematically evaluate the adherence to the post-vaccination observation period and its efficacy in preventing syncope-related injuries.

Another large category of preventable AEFI involves vaccine administration errors of various types. These have been reviewed in the literature [81], and occasionally, serious harm may result from inadvertent substitution of non-vaccine-injectable products that have systemic effects [82]. Other types of mistakes that have been documented include incorrect route of administration and use of adult-formulated vaccines for children and vice versa. Although harm to patients rarely results, such errors can also raise issues of vaccine effectiveness which may require resource-intensive investigation [83]. Providers should adhere to standards for vaccine storage, handling and administration $[28,30]$, assure training of themselves and their staff in proper vaccine usage, and may also wish to participate in local clinic and hospital or broader initiatives designed to decrease medical errors and improve patient safety.

\section{Conclusions}

The vaccine safety issues reviewed in this paper provide concrete examples of how primary care vaccine providers can contribute to enhanced safety of vaccines. For newly licensed vaccines such as rotavirus, attention to new recommendations in combination with reporting of vaccine adverse events to SRS or other designated reporting systems promotes optimal use of these potentially lifesaving products. Clinicians should maintain the awareness of vaccine safety issues, particularly those that have drawn considerable attention from the media, such as thimerosal, and should understand the most current scientific findings and be able to communicate these to concerned parents in a clear and empathic way [84]. The potential for a more widespread use of influenza vaccines, whether or not they are part of a response to an actual or threatened pandemic, means that clinicians should be aware of the safety profile of current influenza vaccines [68] and cooperate with public health authorities on comprehensive local, regional and national pandemic planning efforts. Lastly, in order to minimize the risk of injury to patients of all ages, clinicians should ob- 
serve the most up-to-date recommendations for vaccine storage, handling, administration and follow-up in order to prevent vaccine administration errors and post-vaccination syncope.

Clinical health care providers who are involved in any aspect of vaccine delivery can participate actively in assuring safe vaccination. Expanded clinician participation in vaccine safety reporting systems will improve AEFI surveillance and result in safer vaccines and improved recommendations for their use. A small number of astute reporters to VAERS triggered the withdrawal of the first rotavirus vaccine in 1999 [85], but also began a process that has resulted in new and safer rotavirus vaccines. Providers should report safety concerns to local or national public health authorities through established mechanisms, whether the vaccine involved is newly introduced or established. Another important way that physicians and other experts can improve the safety of vaccines is through participation in the Brighton Collaboration, through working with international colleagues to develop new case definitions or using existing case definitions in trials or post-licensure studies in which they may be participating. Further information is available at http:// www.brightoncollaboration.org. Clinicians should be aware of and regularly access authoritative sources of current vaccine and vaccine safety information, such as the CDC, the WHO and the Vaccine Safety Net websites; patients seeking credible information should also be referred to these sites.

The medical and public health communities across the world will need to continue to comprehensively address vaccine safety concerns in order to maintain high vaccine coverage and reap the full health and economic benefits of vaccination. Ideally, this will involve a coordinated scientific and communication response. Evidencebased elements of vaccine risk communication that providers should be aware of include providing accurate information, maintaining trust in sources of information, keeping open lines of communication with parents, who question use of vaccines for their children, and referring patients to authoritative resources such as websites [86, 87]. Standardizing risk communication of scientific evidence of risk communication about vaccine safety will be an important tool in alleviating or mitigating some of the concerns that can affect the implementation of immunization programs around the world.

\section{References}

$1 \mathrm{http}: / /$ www.thecommunityguide.org/vaccine/vpd-int-prov-standing-orders.pdf.

2 World Bank: World Development Report 1993: Investing in Health. New York, Oxford University Press, 1993.

-3 Centers for Disease Control and Prevention: Ten great public health achievements - United States, 1990-1999. MMWR Morb Mortal Wkly Rep 1999;48:241-243.

4 Bloom DE, Canning D, Weston M: The value of vaccination. World Economics 2005;6:1539.

5 Centers for Disease Control and Prevention: Impact of vaccines universally recommended for children - United States, 1990-1998. MMWR Morb Mortal Wkly Rep 1999;48: 243-248.

6 Folb PI, Bernatowska E, Chen R, et al: A global perspective on vaccine safety and public health: the Global Advisory Committee on Vaccine Safety. Am J Public Health 2004;94:1926-1931.

7 Hinman AR: Public health considerations; in Plotkin SA, Mortimer EA (eds): Vaccine. Philadelphia, Saunders, 1988, pp 587-611.

8 US Office of Technology Assessment: Health Program Advisory Committee, 1981. Cost Effectiveness of Influenza Vaccination. Washington, US Government Printing Office, 1981.
9 Postma MJ, Baltussen RPM, Palache AM, Wilschut JC: Further evidence for favorable cost-effectiveness of elderly influenza vaccination. Expert Rev Pharmacoeconomics Outcome Res 2006;6:215-227.

10 Roush SW, Murphy TV, Vaccine-Preventable Disease Table Working Group: Historical comparisons of morbidity and mortality for vaccine-preventable diseases in the United States. JAMA 2007;298:2155-2163.

11 Centers for Disease Control and Prevention: Progress in global measles control and mortality reduction, 2000-2006. MMWR Morb Mortal Wkly Rep 2007;56:1237-1241.

12 Poehling KA, Talbot TR, Griffin MR, et al: Invasive pneumococcal disease among infants before and after introduction of pneumococcal conjugate vaccine. JAMA 2006; 295:1668-1674.

13 Hutchins SS, Baughman AL, Orr M, et al: Vaccination levels associated with lack of measles transmission among preschool-aged populations in the United States, 1989-1991. J Infect Dis 2004;189:S108-S115.

14 Anderson RM, May RM: Infectious Diseases of Humans: Dynamics and Control. Oxford, Oxford University Press, 1992.

15 Chen RT: Vaccine risks: real, perceived and unknown. Vaccine 1999;17(suppl 3):S41S46.
16 Gangarosa EJ, Galazka AM, Wolfe CR, et al: Impact of anti-vaccine movements on pertussis control: the untold story. Lancet 1998; 351:356-361.

17 Vesikari T, Giaquinto C, Huppertz HI: Clinical trials of rotavirus vaccines in Europe. Pediatr Infect Dis J 2006;25:S42-S47.

18 Vesikari T, Matson DO, Dennehy P, et al: Rotavirus Efficacy and Safety Trial (REST) Study Team. Safety and efficacy of a pentavalent human-bovine (WC3) reassortant rotavirus vaccine. N Engl J Med 2006;354:2333.

19 Ruiz-Palacios GM, Pérez-Schael I, Velázquez FR, et al: Human Rotavirus Vaccine Study Group. Safety and efficacy of an attenuated vaccine against severe rotavirus gastroenteritis. N Engl J Med 2006;354:11-22.

20 Centers for Disease Control and Prevention: Suspension of rotavirus vaccine after reports of intussusception - United States, 1999. MMWR Morb Mortal Wkly Rep 2004;53: 786-789 (erratum published in MMWR Morb Mortal Wkly Rep 2004;53:879).

21 Casey CG, Iskander JK, Roper MH, et al: Adverse events associated with smallpox vaccination in the United States, January-October 2003. JAMA 2005;294:2734-2743. 
-22 Rajante J, Zeller B, Treutiger I, Rosthoj S: NOPHO ITP working group and five national study groups. Vaccination associated thrombocytopenic purpura in children. Vaccine 2007;25:1838-1840.

23 Arness MK, Eckart RE, Love SS, et al: Myopericarditis following smallpox vaccination. Am J Epidemiol 2004;160:642-651.

24 Ellenberg SS, Foulkes MA, Midthun K, Goldenthal KL: Evaluating the safety of new vaccines: summary of a workshop. Am J Public Health 2005;95:800-807.

25 Izurieta HS, Haber P, Wise RP, et al: Adverse events reported following live, cold-adapted, intranasal influenza vaccine. JAMA 2005; 294:2720-2725 (erratum published in JAMA 2005;294:3092).

26 Halsey N: The science of evaluation of adverse events associated with vaccination. Semin Pediatr Infect Dis 2002;13:205-214.

-27 Destefano F, Vaccine Safety Datalink Research Group: The Vaccine Safety Datalink project. Pharmacoepidemiol Drug Saf 2001; 10:403-406.

28 Kroger AT, Atkinson WL, Marcuse EK, Pickering LK: Advisory Committee on Immunization Practices (ACIP), Centers for Disease Control and Prevention. General recommendations on immunization: recommendations of the Advisory Committee on Immunization Practices (ACIP). MMWR Recomm Rep 2006;55:1-48.

$29 \mathrm{http}: / /$ www.cdc.gov/nip/acip.

30 http://www.who.int/vaccine_safety/en/.

31 http://www.iom.edu/.

32 Immunization Safety Review: Hepatitis B Vaccine and Demyelinating Neurological Disorders. Washington, Board on Health Promotion and Disease Prevention (HPDP), Institute of Medicine (IOM), 2002. http:// www.iom.edu/CMS/3793/4705/4435.aspx.

33 http://www.cochrane.org/.

- 34 Peter G, Myers MG, National Vaccine Advisory Committee, National Vaccine Program Office: Intussusception, rotavirus, and oral vaccines: summary of a workshop. Pediatrics 2002;110:e67.

35 World Health Organization: Surveillance of adverse events following immunization. Field guide for managers of immunization programmes. Global Programme for Vaccines and Immunization. Expanded Programme on Immunisation. Geneva, World Health Organization, 1997. http://who.int/ vaccines-documents/DocsPDF/www9541. pdf.

36 Ball R, Halsey N, Braun MM, et al: Development of case definitions for acute encephalopathy, encephalitis, and multiple sclerosis reports to the Vaccine Adverse Event Reporting System. J Clin Epidemiol 2002;55: 819-824.

37 Kohl KS, Gidudu J, Bonhoeffer J, et al: The development of standardized case definitions and guidelines for adverse events following immunization. Vaccine 2007;25: 5671-5674.
38 Kohl KS, Bonhoeffer J, Braun MM, et al: The Brighton Collaboration: Creating a global standard for case definitions (and guidelines) for adverse events following immunization; in AHRQ: Advances in Patient Safety. Concepts and Methodology. Rockville, AHRQ, 2005, vol 2, pp 87-102.

39 Bonhoeffer J, Kohl K, Chen R, et al: The Brighton Collaboration-enhancing vaccine safety. Vaccine 2004;22:2046.

40 Bonhoeffer J, Heininger U, Kohl K, et al: Standardized case definitions of adverse events following immunization (AEFI). Vaccine 2004;22:547-550.

41 Kohl KS, Bonhoeffer J, Chen RT, et al: The Brighton Collaboration: enhancing comparability of vaccine safety data. ISPE commentary. Pharmacoepidemiol Drug Saf 2003;12:335-340.

42 Bonhoeffer J, Kohl K, Chen R, et al: The Brighton Collaboration: addressing the need for standardized case definitions of adverse events following immunization (AEFI). Vaccine 2002;21:298-302.

43 Marcy SM, Kohl KS, Dagan R, et al: The Brighton Collaboration Fever Working Group. Fever as an adverse event following immunization: case definition and guidelines of data collection, analysis, and presentation. Vaccine 2004;22:551-556.

44 Beigel J, Kohl KS, Khuri-Bulos N, et al: Rash including mucosal involvement: case definition and guidelines for collection, analysis, and presentation of immunization safety data. Vaccine 2007;25:5697-5706.

45 Tapiainen T, Prevots R, Izurieta HS, et al: Aseptic meningitis: case definition and guidelines for collection, analysis and presentation of immunization safety data. Vaccine 2007;25:5793-5802.

46 Bines JE, Kohl KS, Forster J, et al: The Brighton Collaboration Intussusception Working Group. Acute intussusception in infants and children as an adverse event following immunization: case definition and guidelines of data collection, analysis, and presentation. Vaccine 2004;22:569-574.

47 World Health Organization: WHO consultation on global monitoring of adverse events following immunization, 9-10 January 2006. Wkly Epidemiol Rec 2006;81:261-272.

48 Food and Drug Administration: Guidance for industry. Toxicity grading scale for healthy adult and adolescent volunteers enrolled in preventive vaccine clinical trials. http://www.fda.gov/cber/guidelines. htm\#toxvac.

49 European Medicines Agency: Note for guidance on the clinical evaluation of vaccines. London, EMEA, Committee for Human Medicinal Products (CHMP), 2005. http://www. emea.eu.int/pdfs/human/vwp/16465305en. pdf.
50 International Conference on Harmonisation of Technical Requirements for Registration of Pharmaceuticals for Human Use (ICH). Guidelines for clinical safety assessment (E2a-e). http://www.ich.org/ (accessed December 13, 2007).

51 Yokotsuka M: The use of a medical dictionary for regulatory activities terminology (MedDRA) in prescription-event monitoring in Japan (J-PEM). Int J Med Inform 2000; 57:139-153.

52 Moher D, Cook DJ, Eastwood S, et al: Improving the quality of reports of meta-analyses of randomised controlled trials: the QUOROM statement. Quality of Reporting of Meta-analyses. Lancet 1999;354:18961900. http://www.consort-statement.org/ Initiatives/complements.htm (accessed December 13, 2007).

53 Hviid A: Postlicensure epidemiology of childhood vaccination: the Danish experience. Expert Rev Vaccines 2006;5:641-649.

54 Marshall HS, Gold MS, Gent R, et al: Ultrasound examination of extensive limb swelling reactions after diphtheria-tetanus-acellular pertussis or reduced-antigen content diphtheria-tetanus-acellular pertussis immunization in preschool-aged children. Pediatrics 2006;118:1501-1509.

55 Zanoni G, Ferro A, Valsecchi M, Tridente G: The 'Green Channel' of the Veneto region as a model for vaccine safety monitoring in Italy. Vaccine 2005;23:2354-2358.

56 Gold M, Goodwin H, Botham S, et al: Revaccination of 421 children with a past history of an adverse vaccine reaction in a special immunisation service. Arch Dis Child 2000;83:128-131.

57 Klein NP, Fireman B, Enright A, et al: Clinical Immunization Safety Assessment Network. A role for genetics in the immune response to the varicella vaccine. Pediatr Infect Dis J 2007;26:300-305.

58 http://www.cdc.gov/od/science/iso/research activties/cisa.htm.

59 Centers for Disease Control and Prevention: Rotavirus vaccine for the prevention of rotavirus gastroenteritis among children. MMWR Morb Mortal Wkly Rep 1999;48:120.

60 Centers for Disease Control and Prevention: Withdrawal of rotavirus vaccine recommendation. MMWR Morb Mortal Wkly Rep 1999;48:1007.

-61 Centers for Disease Control and Prevention: Postmarketing monitoring of intussusception after RotaTeq vaccination - United States, February 1, 2006-February 15, 2007. MMWR Morb Mortal Wkly Rep 2007;56: 218-222.

62 Matsuno S, Utagawa E, Sugiura A: Association of rotavirus infection with Kawasaki syndrome. J Infect Dis 1983;148:177.

63 Thimerosal in vaccines: a joint statement of the American Academy of Pediatrics and the Public Health Service. Neonatal Netw 1999; 18:65, 72 . 
64 Dayan GH, Iskander J, Glasser J, et al: Tracking vaccine lot lifecycles using reports to the vaccine adverse event reporting system (VAERS). Pharmacoepidemiol Drug Saf 2005; 14:671-676.

65 Meadows M: IOM report: no link between vaccines and autism. FDA Consum 2004;38: $18-19$.

66 DeStefano F: Vaccines and autism: evidence does not support a causal association. Clin Pharmacol Ther 2007;82:756-759.

-67 Thompson WW, Price C, Goodson B, et al: Vaccine Safety Datalink Team. Early thimerosal exposure and neuropsychological outcomes at 7 to 10 years. N Engl J Med 2007; 357:1281-1292.

68 Fiore AE, Shay DK, Haber P, et al: Advisory Committee on Immunization Practices (ACIP), Centers for Disease Control and Prevention (CDC). Prevention and control of influenza. Recommendations of the Advisory Committee on Immunization Practices (ACIP), 2007. MMWR Recomm Rep 2007; 56:1-54.

69 Wakefield AJ, Murch SH, Anthony A, et al: Ileal-lymphoid-nodular hyperplasia, nonspecific colitis, and pervasive developmental disorder in children. Lancet 1998;351:637641.

70 Ashraf H: US expert group rejects link between MMR and autism. Lancet 2001;357: 1341.
71 DeStefano F, Thompson WW: MMR vaccine and autism: an update of the scientific evidence. Expert Rev Vaccines 2004;3:19-22.

72 Murch SH, Anthony A, Casson DH, et al: Partial retraction. Lancet 2004;363:750.

73 Seasonal and Pandemic Influenza 2007 report: Seasonal and Pandemic Influenza Meeting. American Academy of Pediatrics (AAP), the Infectious Disease Society of America (IDSA), and the Society of Healthcare Epidemiology of America (SHEA) with participation by the Centers for Disease Control and Prevention (CDC). http:// influenza-info.org/cme/2007/monograph/ monograph.pdf.

74 Food and Drug Administration: VRBPAC briefing materials from February 27, 2007, meeting. www.fda.gov (accessed August 4, 2007).

75 Iskander J, Haber P, Herrera G. Monitoring vaccine safety during an influenza pandemic. Yale J Biol Med 2005;78:265-275.

76 Centers for Disease Control and Prevention: Pandemic influenza vaccine plan, chapter 8 . December 2007. Unpublished.

77 Oster LG, Sterner U, Lindahl IL: Physiologic responses in blood phobics. Behav Res Ther 1984;22:109-117.

78 Chen RT, Rastogi SC, Mullen JR, et al: The Vaccine Adverse Event Reporting System (VAERS). Vaccine 1994;12:542-550.

79 Braun MM, Patriarca PA, Ellenberg SS: Syncope after immunization. Arch Pediatr Adolesc Med 1997;151:255-259.
80 Woo EJ, Ball R, Braun MM: Fatal syncoperelated fall after immunization. Arch Pediatr Adolesc Med 2005;159:1083.

81 Varricchio F, Reed J, VAERS Working Group: Follow-up study of medication errors reported to the vaccine adverse event reporting system (VAERS). South Med J 2006;99:486489.

82 Varricchio F: Medication errors reported to the vaccine adverse event reporting system (VAERS). Vaccine 2002;20:3049-3051.

83 Centers for Disease Control and Prevention: Inadvertent misadministration of meningococcal conjugate vaccine - United States, June-August 2005. MMWR Morb Mortal Wkly Rep 2006;55:1016-1017.

84 Smith PJ, Kennedy AM, Wooten K, et al: Association between health care providers' influence on parents who have concerns about vaccine safety and vaccination coverage. $\mathrm{Pe}$ diatrics 2006;118:e1287-e1292.

85 Zanardi LR, Haber P, Mootrey GT, et al: Intussusception among recipients of rotavirus vaccine: reports to the vaccine adverse event reporting system. Pediatrics 2001;107:E97.

86 Hilton S, Petticrew M, Hunt K: Parents' champions vs vested interests: who do parents believe about MMR? A qualitative study. BMC Public Health 2007;7:42.

87 Tenrreiro KN: Time-efficient strategies to ensure vaccine risk/benefit communication. J Pediatr Nurs 2005;20:469-476. 